\title{
UBICACIÓN ÓPTIMA DE MÓDULOS ELECTORALES EN EL ESTADO DE CHIHUAHUA (MÉXICO) UTILIZANDO MODELOS DE LOCALIZACIÓN- ASIGNACIÓN MEDIANTE UN SIG ${ }^{1}$
}

\author{
José María Casado Izquierdo \\ Instituto de Geografía. Universidad Nacional Autónoma de México \\ chema@igg.unam.mx \\ Celia Palacios Mora \\ Instituto Federal Electoral \\ celia.palacios@ife.org.mx
}

\section{RESUMEN}

Este artículo ofrece una propuesta de ubicación de módulos de atención ciudadana fijos a partir de la aplicación de un modelo de localización-asignación utilizando un Sistema de Información Geográfica (SIG). Esta propuesta de ubicación se realiza a nivel de sección electoral tomando al estado de Chihuahua como un caso piloto para la posible réplica de este análisis al resto de entidades federativas de México.

Palabras clave: localización óptima, modelos de localización-asignación, geografía comercial.

\section{ABSTRACT}

This paper offers a proposal for the location of fixed service modules for citizens using a location-allocation model implemented in a Geographic Information System (GIS). Locations are proposed at the level of electoral sections, taking the state of Chihuahua as a test case for possible replication of this analysis to other Mexican states.

Key words: optimal location, location-allocation models, business geography.

Fecha de recepción: febrero 2011.

Fecha de aceptación: julio 2012.

1 Investigación desarrollada en el marco del Comité Técnico del Padrón Electoral del Instituto Federal Electoral. 


\section{INTRODUCCIÓN}

Los módulos de atención ciudadana desempeñan un papel fundamental dentro de las funciones del Instituto Federal Electoral (IFE) como organismo a cargo de organizar las elecciones federales, al ser los encargados de proporcionar la credencial de elector a las personas que cumplen con los requisitos exigidos para su adquisición, esto es, que hubiesen:

- Alcanzado la ciudadanía en tiempo reciente.

- Modificado su domicilio.

- Extraviado su credencial.

- Sido rehabilitados después de haber sido suspendidos en sus derechos políticos.

Cabe mencionar que la credencial de elector no sólo es un documento imprescindible para poder ejercer el derecho al voto en las distintas elecciones, sino que, en la práctica, y ante la ausencia de un identificación oficial obligatoria, es el medio de identificación más difundido en México, siendo requerida para la realización de diversos trámites ante instituciones tanto públicas como privadas (p.e, la apertura de una cuenta bancaria). ${ }^{2}$

La necesidad de garantizar el derecho al voto a todos los ciudadanos y, por tanto, el acceso a la credencial de elector, ha llevado al IFE a desarrollar una estrategia bien definida en lo que se refiere a los módulos de atención ciudadana, estrategia que ha derivado en el establecimiento de tres tipos de módulos a fin de atender distintas situaciones: fijos, semifijos y móviles. La ubicación y uso de estos distintos tipos responde principalmente a la distribución de la población:

- Los módulos fijos se localizan básicamente en grandes ciudades a fin de cubrir la alta demanda que generan estas grandes concentraciones de población, situación especialmente relevante en México donde el sistema urbano se caracteriza por su macrocefalia. También suelen ubicarse en ciertas cabeceras distritales de mediano tamaño a fin de cubrir su demanda y la de áreas aledañas.

- Los módulos móviles cubren la demanda de extensas áreas rurales donde la población presenta una distribución muy dispersa.

- Por último, los módulos semifijos se utilizan tanto para atender la demanda en áreas con población dispersa como para realizar campañas de credencialización en ciertas zonas al interior de las ciudades.

Punto relevante en la función de estos módulos es, obviamente, su productividad, la cual depende de diversos factores:

- Tamaño, generalmente evaluado en función del número de computadoras de que disponen.

- Horario: deben cubrir un horario de 7 horas de lunes a viernes y de 5 horas los sábados; sin embargo, existen módulos con doble turno, es decir, con un horario de 11 horas de lunes a viernes y de 5 horas los sábados.

2 La creación, todavía en vías, de una cédula de identidad ciudadana por parte del Registro Nacional de Población (RENAPO) ha suscitado diversas fricciones entre esta institución, dependiente de la Secretaría de Gobernación, y el propio IFE ante la posibilidad de utilizar la base de datos de credenciales electorales del IFE como uno de los puntos de partida para la elaboración de dicha cédula de identidad; ello debido a cuestiones de confidencialidad, posible uso indebido y rivalidad de ambos instrumentos como medios de identificación (Zebadúa, 2010). 
- Promoción y conocimiento de su ubicación por parte de los ciudadanos. Este hecho suele estar vinculado con la antigüedad del propio módulo, por lo que su relocalización pudiera ocasionar una disminución de los usuarios en el corto plazo.

- Ubicación, y por tanto accesibilidad por parte de los ciudadanos.

Es en este último punto donde este estudio pretende realizar una aportación relevante en cuanto a módulos fijos, dado que su ubicación actual no responde a ninguna estrategia oficial y/o predefinida de localización, sino que su distribución ha sido realizada de manera «intuitiva» y respondiendo a criterios no explícitos ni uniformes en el territorio nacional.

Es claro que la provisión de módulos fijos supone para el IFE una fuerte inversión en términos de infraestructura (desde la adquisición de equipo de cómputo hasta pago de salarios o de rentas de alquiler de locales), por lo que su eficiencia/productividad debería ser un criterio relevante a la hora de decidir su localización. No obstante, el hecho de que ciertos módulos se asienten en locales cedidos por otras instituciones también tiene un impacto desde el punto de vista de su costo. Por otro lado, los propios partidos políticos son conscientes de las posibles implicaciones de la reubicación de los módulos fijos en términos de facilitar/dificultar el acceso a la credencial de elector de sus potenciales votantes, por lo que dicha reubicación no estaría exenta de presiones políticas.

\section{ESTRUCTURA GEO-ELECTORAL DE CHIHUAHUA}

El análisis desarrollado tiene como objeto de estudio el estado de Chihuahua, entidad elegida por coincidir en ella una serie de características que la hacen interesante como caso piloto:

- De acuerdo con IFE-RFE (2005) Chihuahua presenta una gran diversidad y complejidad en sus distritos electorales, coexistiendo:

o Grandes concentraciones urbanas, donde destacan las ciudades de Chihuahua y sobre todo Ciudad Juárez, la cual ha venido experimentando un crecimiento explosivo en los últimos años, con un incremento de más de 500 mil habitantes y una tasa de crecimiento del $3.37 \%$ entre 1990 y 2005 (SEDESOL et. al. 2007).

o Extensas áreas rurales/desérticas con población muy dispersa.

- Alta demanda potencial de credenciales en ciertos distritos de la entidad, entendiéndose como demanda potencial la diferencia entre la población de 18 años y más residente en la entidad y la población que cuenta con una credencial electoral actualizada. Esta importante demanda potencial viene dada por las altas tasas de inmigración generadas por la implantación y desarrollo de la industria maquiladora de exportación y por ser Ciudad Juárez una destacada zona de tránsito para quienes buscan cruzar la frontera hacia Estados Unidos. Si bien estos inmigrantes pueden o no contar con credencial, existe, al menos, la necesidad de actualizar su domicilio a fin de poder ejercer su derecho al voto en la circunscripción electoral donde residen actualmente. ${ }^{3}$

3 De acuerdo con IFE-RFE (2008) el cambio de domicilio constituye el mayor problema de actualización del padrón electoral, pese a que de acuerdo al Art. 175 del Código Federal de Instituciones y Procedimientos Electorales (COFIPE) «Los ciudadanos están obligados ... a informar a éste [Registro Federal de Electores] de su cambio de domicilio dentro de los treinta días siguientes a que éste ocurra». 
De acuerdo con IFE-RFE (2008a) en el estado de Chihuahua el 35.2\% de la población mayor de 18 años requiere de tramitar una credencial para votar, lo que sitúa a esta entidad en séptimo lugar tras Baja California Sur (47.4\%), Quintana Roo (42.0\%), Baja California (39.4\%), Guerrero (36.0\%), Colima (35.9\%) y Jalisco (35.5\%). Este porcentaje representa, para el caso de Chihuahua, un total estimado de 750 mil ciudadanos, cifra nada desdeñable desde el punto de vista de sus posibles implicaciones electorales.

A nivel distrito este porcentaje varía de $31.02 \%$ a $43.36 \%$, siendo mayores los porcentajes en la zona norte y centro del estado, donde se asientan las ciudades de Chihuahua y Ciudad Juárez respectivamente. Este problema se acentúa en el distrito 0801, situación que se explica por la expansión de la mancha urbana de Ciudad Juárez desde los distritos 0802, 0803 y 0804 sobre dicho distrito 0801 (véase Figura 2). Así, pese a su gran extensión territorial, la demanda potencial existente en el distrito 0801 se localizaría fundamentalmente en las vecindades de los distritos arriba mencionados. Este porcentaje de demanda potencial del distrito 0801 es incluso uno de los más altos a nivel nacional, comparable al de distritos donde se ubican algunos de los municipios de mayor crecimiento demográfico del país, caso de Ixtapaluca en el estado de México, Los Cabos en Baja California Sur o Benito Juárez (Cancún) en Quintana Roo.

Desde el punto de vista de la estructura geo-electoral la entidad se encuentra organizada en ocho distritos y 2,909 secciones federales, contando para 2009 con 40 módulos con las siguientes características (Tabla 1):

- 22 fijos, de los cuales 4 son de doble turno, con un total de 76 computadoras y una productividad de 11.7 mil trámites realizados por cada computadora entre julio 2006enero 2009;

- 2 semifijos, con 3 computadoras y una productividad de 4.8 mil trámites; y

- 16 móviles, con 21 computadoras y una productividad de 8.8 mil trámites.

Tabla 1

MÓDULOS DE ATENCIÓN CIUDADANA FIJOS EN CHIHUAHUA, 2009

\begin{tabular}{|c|c|c|c|c|c|}
\hline \multirow{2}{*}{ Área urbana } & \multirow{2}{*}{ Distrito } & \multicolumn{2}{|c|}{ Módulos fijos } & \multirow{2}{*}{ Computadoras } & \multirow{2}{*}{$\begin{array}{l}\text { Padrón electoral } \\
\text { (Ciudadanos) }\end{array}$} \\
\hline & & Total & Doble turno & & \\
\hline \multirow[t]{4}{*}{ Cd. Juárez } & 0801 & 4 & - & 16 & 290,331 \\
\hline & 0802 & 3 & 1 & 15 & 234,746 \\
\hline & 0803 & 3 & - & 7 & 274,949 \\
\hline & 0804 & 3 & 1 & 10 & 243,594 \\
\hline \multirow[t]{2}{*}{ Chihuahua } & 0806 & 2 & 1 & 7 & 289,180 \\
\hline & 0808 & 3 & 1 & 10 & 288,801 \\
\hline Cuauhtémoc & 0807 & 1 & - & 3 & 80,530 \\
\hline Delicias & 0805 & 1 & - & 3 & 85,890 \\
\hline Hidalgo del Parral & 0809 & 1 & - & 3 & 78,359 \\
\hline Nvo. Casas Grandes & 0807 & 1 & - & 2 & 71,112 \\
\hline Resto del estado: & & 0 & - & 0 & 493,426 \\
\hline TOTAL: & & 22 & 4 & 76 & $2,400,918$ \\
\hline
\end{tabular}

Fuente: Elaboración propia a partir de IFE-RFE (2009a) 
De entre los diez módulos con mayor productividad, dos son módulos móviles que realizaron recorridos en los distritos 0809 y 0807, ubicándose en primer lugar el módulo fijo de doble turno 080223 ubicado en Cd. Juárez, con una productividad de 19 mil trámites.

Respecto a la distribución geográfica de los módulos fijos, éstos se sitúan en las principales áreas urbanas de la entidad, destacando Ciudad Juárez con 13 módulos fijos y 48 computadoras, lo que supone en torno al 59 y $63 \%$ de dicha infraestructura (Tabla 1). Este porcentaje puede considerarse como excesivo dado que en los módulos fijos de Cd. Juárez se procesaron únicamente el $53 \%$ de los trámites realizados en este tipo de módulos en toda la entidad, indicativo de una cierta inequidad en su distribución a favor de Cd. Juárez.

\section{MODELOS DE LOCALIZACIÓN EN EL CAMPO GEOGRÁFICO. LOS MODELOS DE LOCALI- ZACIÓN-ASIGNACIÓN}

El problema de la localización de una determinada instalación o servicio en el espacio, en este caso módulos fijos, constituye uno de los planteamientos básicos en la Geografía (Haggett, 1965). Dicho problema surge, básicamente, por la denominada fricción de la distancia o impedancia, esto es, el costo de desplazar un recurso de un lugar a otro.

Tradicionalmente el concepto de fricción de la distancia ha sido interpretado, de manera operativa, a través de distintas variables: la distancia entre dos puntos, el tiempo de traslado o el costo de dicho traslado, entre otros. Tras estos simples planteamientos se encuentran, sin embargo, complejas y múltiples tomas de decisiones, por ejemplo, cuándo, cómo y a dónde desplazarse o dónde localizarse (desde el propio lugar de residencia hasta la ubicación de una instalación).

La importancia de la fricción de la distancia en el análisis locacional tiene su antecedente más relevante en el modelo agrícola de círculos concéntricos elaborado por Thünen (1826); en este modelo, los costes del transporte al mercado central determinan, junto con la distinta productividad de los cultivos, una renta marginal decreciente de la tierra a medida que se aleja del mercado central. Esta importancia de los costos de transportes sería posteriormente incorporada en la localización industrial por Weber (1909) y en la teoría de los lugares centrales de Christaller (1933), quien desarrolla conceptos como centralidad, umbral y alcance para explicar la distribución territorial y jerárquica de los asentamientos como centros proveedores de servicios. Dichos antecedentes serán recogidos y desarrollados por autores posteriores como Lösch (1940), quien amplía la teoría de Christaller, Ullman (1941), quien introduce la teoría de lugares centrales en la geografía estadounidense, Isard (1956, 1960), padre de la Ciencia Regional, e incluso por Alonso (1964) en su teoría urbana de la localización económica.

A su vez, el análisis de las interacciones espaciales tendrá como punto de partida el modelo gravitacional de Newton, adaptado por Reilly en 1929/1931 para el análisis del comercio al por menor, dando lugar con el tiempo a lo que se han venido a denominar modelos potenciales de interacción (Isard, 1998:248), de amplio uso en problemas de delimitación de áreas de mercado y, en consecuencia, de problemas de ubicación de instalaciones (Tabla 2). Si bien el término áreas de mercado sugiere su aplicación únicamente desde un punto de vista de instalaciones privadas, también se ha aplicado a numerosos equipamientos públicos urbanos como hospitales, escuelas, oficinas postales o áreas recreativas, entre otras, definién- 
dose como el área geográfica desde la cual una instalación o equipamiento atrae a la mayoría de sus usuarios.

Tabla 2

MODELOS GRAVITACIONALES YALGUNAS DERIVACIONES

\begin{tabular}{|c|c|}
\hline $\begin{array}{l}\text { A. Modelo gravitacional de Newton } \\
F_{i j}=G \frac{m_{i} * m_{j}}{d_{i j}^{2}} \\
\mathrm{~F}_{\mathrm{ij}}=\text { Fuerza de atracción entre los cuerpos } \\
\text { i y j. } \\
\mathrm{G}=\text { Constante de gravitación universal (en } \\
\text { la Tierra } 9.8 \mathrm{~m} / \mathrm{s}^{2} \text { ) } \\
\mathrm{m}_{\mathrm{i},} \mathrm{m}_{\mathrm{j}}=\text { Masa del cuerpo i y j } \\
\text { respectivamente. } \\
\mathrm{d}_{\mathrm{ij}}=\text { Distancia entre los cuerpos i y j. }\end{array}$ & $\begin{array}{l}\text { B. Modelo de comercio al por menor de } \\
\text { Reilly }(1929,1931) \\
\frac{V_{i}}{V_{j}}=\left(\frac{S_{i}}{S_{j}}\right) *\left(\frac{d_{j t}}{d_{i t}}\right)^{2} \\
\mathrm{~V}_{\mathrm{i}}, \mathrm{V}_{\mathrm{j}}=\text { Ventas en i y j respectivamente } \\
\text { atraídas desde un punto intermedio } \mathrm{t} \text {. } \\
\mathrm{S}_{\mathrm{i}}, \mathrm{S}_{\mathrm{j}}=\text { Superficie comercial/«masa» en i y } \mathrm{j} \\
\text { respectivamente. } \\
\mathrm{d}_{\mathrm{it}}, \mathrm{d}_{\mathrm{jt}}=\text { Distancia entre i y } \mathrm{t} \text { y entre } \mathrm{j} \mathrm{y} \mathrm{t} \\
\text { respectivamente. }\end{array}$ \\
\hline $\begin{array}{l}\text { C. Punto/distancia de indiferencia derivado } \\
\text { del modelo de Reilly (Converse, 1949) } \\
d_{j t}=\frac{d_{i j}}{1+\sqrt{\frac{S_{i}}{S_{j}}}} \\
\mathrm{~d}_{\mathrm{jt}}=\text { Distancia de } \mathrm{j} \text { al punto de indiferencia } \\
\mathrm{t} . \\
\mathrm{d}_{\mathrm{ij}}=\text { Distancia entre i y j (centros } \\
\text { comerciales en competencia). } \\
\mathrm{S}_{\mathrm{i}}, \mathrm{S}_{\mathrm{j}}=\text { Superficie comercial/«masa» en i y j } \\
\text { respectivamente. }\end{array}$ & $\begin{array}{l}\text { D. Modelo probabilístico de comercio al } \\
\text { por menor de Huff }(1963,1964) \\
p_{i j}=\frac{S_{j} * t_{i j}^{\lambda}}{\sum_{j}^{n} \frac{S_{j}}{t_{i j}^{\lambda}}} \\
\mathrm{p}_{\mathrm{ij}}=\text { Probabilidad de que un comprador en } \mathrm{i} \\
\text { se desplace a comprar en } \mathrm{j} \text {. } \\
\mathrm{S}_{\mathrm{j}}=\text { Superficie comercial/«masa» en } \mathrm{j} \text {. } \\
\mathrm{t}_{\mathrm{ij}}=\text { Tiempo de desplazamiento entre } \mathrm{i} \mathrm{y} \text {. } \\
\mathrm{l}=\text { Exponente a ser calibrado a partir de } \\
\text { observaciones empíricas, representando la } \\
\text { sensibilidad del consumidor hacia el tiempo } \\
\text { de desplazamiento. } \\
\mathrm{n}=\text { Total de ubicaciones comerciales que } \\
\text { pueden ser accedidas por un comprador en } \\
\mathrm{i} .\end{array}$ \\
\hline
\end{tabular}

La creciente generalización del modelo gravitacional implicó la consideración de diversos elementos representativos de las masas planetarias de Newton (población, superficie comercial, empleos, PIB, ..), hasta quedar finalmente abstraídas como factores de atracción (del lugar de destino) y expulsión (del lugar de origen). Mientras, la fricción de la distancia, en principio expresada en términos de unidades lineales, quedará generalizada como impedancia o conjunto de factores que dificultan el desplazamiento (teoría general del movimiento de Alonso). 
La evolución de los modelos gravitacionales hacia modelos probabilísticos o estocásticos conducirá al desarrollo de modelos maximizadores de entropía donde destaca la obra de Wilson (Roy, 2004: 14-19; 87-91) y la obtención de modelos de interacción restringidos (en origen, en destino o en ambos).

Con el tiempo, otros factores serán incluidos en los modelos probabilísticos, por ejemplo, la restricción impuesta por el costo de transporte, la posibilidad de desplazamientos multipropósito, la competencia entre diferentes posibles destinos (intervening opportunities) o el uso de modelos desagregados vs. modelos agregados a fin de considerar el carácter heterogéneo tanto de los diferentes orígenes como destinos: distinta «predisposición» a desplazarse de los diferentes individuos en función de sus características o distinta «atracción» de una misma variable, por ejemplo superficie comercial, en función de otras variables complementarias (diversidad de productos ofrecidos, costos de los mismos, etc.).

En definitiva, el desarrollo de los diferentes modelos de interacción espacial ha estado vinculado al progreso de técnicas estadísticas principalmente relacionadas con la estadística inferencial, en particular con técnicas de regresión y probabilísticas, tendiendo con el tiempo a integrarse en la creciente preocupación por el espacio por parte de la Economía y el subsiguiente florecimiento de la econometría espacial, ambas favorecidas por el incremento de la capacidad de cómputo, la mayor cantidad de información disponible y el desarrollo de los Sistemas de Información Geográfica (SIG). Sobresale también el amplio uso de la programación lineal como técnica optimizadora que busca maximizar o minimizar una función objetivo de acuerdo con una serie de restricciones (Wang, 2006:189).

Dentro de los modelos de localización un subconjunto está representado por los denominados modelos de localización-asignación (location-allocation models), los cuales se preocupan por dónde ubicar una instalación y que parte de la demanda debería ser atendida desde cada instalación. Su objetivo es localizar una o más instalaciones de manera que pueda satisfacer la demanda de la manera más eficiente, considerando que la demanda se encuentra dispersa en el espacio y que la oferta no puede ubicarse en todos los lugares sino únicamente en un número limitado de localizaciones.

De acuerdo a la taxonomía establecida por Daskin (2008:283-284) se diferencian cuatro tipos de modelos de localización:

1. Modelos analíticos, donde se asume que la demanda se distribuye de una determinada manera (por ejemplo de manera uniforme) en el interior de un área, pudiendo ubicarse la oferta (instalación) en cualquier ubicación al interior de dicha área.

2. Modelos continuos, donde, a diferencia de los modelos analíticos, se asume que la demanda y la oferta se ubican en puntos específicos, en puntos discretos.

3. Modelos de red, donde la demanda y la oferta se ubican sólo en una red.

4. Modelos discretos, donde la distancia o coste entre cualquier par de localizaciones puede ser arbitraria, aunque generalmente siguen algún tipo de regla (por ejemplo, euclidiana, Manhattan o a través de una red).

a. Modelos basados en la cobertura, que asumen que la demanda debe ser cubierta dentro del rango de una determinada distancia.

b. Modelos basados en la mediana (punto mediano), que buscan minimizar la distancia promedio ponderada por la demanda considerando la ubicación de la demanda y la instalación a la que es asignada. 
c. Otros.

Dentro de los modelos discretos algunos de los problemas clásicos de localización serían (Miller y Shaw, 2001:199-209; Moreno, 2004:53-101; Wang, 2006:200-202; Buzai y Baxendale, 2008: 100-102; Daskin, 2008):

- El modelo p-mediano: ubicar un número prefijado de instalaciones minimizando la distancia total entre los puntos de demanda y dichas instalaciones, ponderándose la distancia entre cada punto de demanda y la instalación en función de dicha demanda; todos los puntos de demanda son asignados a la instalación más próxima.

- Modelo de localización y cobertura (location set covering problem-LSCP): determinar el número y localización de instalaciones necesarias para cubrir la totalidad de la demanda.

- Modelo de máxima cobertura (maximal covering location problem-MCLP): situar un número predeterminado de instalaciones maximizando la demanda atendida/cobertura, considerando para ello un desplazamiento máximo prefijado (Church y Revelle, 1974).

- Modelo p-centro: ubicar un número predeterminado de instalaciones minimizando la distancia máxima a recorrer desde los puntos de demanda; todos los puntos de demanda son asignados.

Mientras estos modelos responden a la localización de instalaciones y servicios donde la proximidad a la población es deseable, en el extremo opuesto se sitúan aquellos modelos que tratan de responder a la ubicación de instalaciones no deseables, por ejemplo depósitos de residuos sólidos urbanos, plantas de tratamiento de aguas residuales o depósitos de residuos peligrosos. Su ubicación óptima, siguiendo a Bosque et al. (2006:55-56) debe responder a dos criterios básicos: eficiencia espacial, esto es, mayor lejanía posible a la población potencialmente afectada pero a la vez mayor proximidad posible a sus usuarios (por ejemplo, los generadores de residuos peligrosos); y justicia espacial, es decir, la no concentración de este tipo de instalaciones en una determinada zona.

Además de los mencionados, otros problemas de localización más complejos han sido planteados: la relación entre localización de instalaciones y rutas de distribución, el emplazamiento de centros de concentración y transbordo de carga (hubs), la ubicación de instalaciones cuya implantación tiene como fin servir/interceptar una demanda en movimiento (gasolineras, señales de tránsito, ...), entre otros (Smith et al., 2009).

\section{IMPLEMENTACIÓN DE DIFERENTES MODELOS DE LOCALIZACIÓN-ASIGNACIÓN}

En la implementación de los modelos de localización en un SIG diversos aspectos deben ser considerados. Siguiendo a Bosque y Moreno (2004:11-12) los pasos a desarrollar serían:

1. Planteamiento del problema: identificar la ubicación de módulos fijos a nivel sección electoral.

2. Criterios a optimizar: respecto a este apartado se debe señalar que existe un importante debate en la ubicación de instalaciones, sobre si su objetivo debe ser la eficiencia o la equidad espacial, dado que la desigualdad en el acceso a determinados servicios implica que parte de la población quede marginada del acceso a estos servicios o que dicho acceso le sea más difícil/oneroso (Garrocho, 1993; Bosque y Moreno, 2007). 
3. Fijación de las restricciones:

3.1. Restricción espacial: el análisis se limita al estado de Chihuahua como ejemplo de su posible aplicación al resto de entidades federativas del país.

3.2. Restricción desde la oferta:

3.2.1. Del total de 2,909 secciones se consideran como posibles candidatas para la ubicación de un módulo fijo las siguientes: 2,157 secciones correspondientes a localidades urbanas y 52 secciones adicionales que presentan una demanda igual o superior a 500 ciudadanos. Ello supone el $75.9 \%$ de todas las secciones con el $94.7 \%$ de la demanda. Esta restricción tiene como objeto evitar la localización de un módulo fijo en secciones con muy baja demanda pero estratégicamente emplazadas a una distancia/ tiempo equidistante de secciones con una demanda relevante. Esta decisión se adoptó a posteriori tras comprobar la situación descrita, aunque como se verá esta circunstancia continua produciéndose en cierta medida.

3.2.2. El número de módulos fijos a ubicar es de 22, al ser ésta la cifra de módulos fijos existentes.

3.3. Restricción desde la demanda: a fin de fijar la demanda existente se utiliza la base de datos de solicitudes exitosas (IFE-RFE, 2009b), la cual refleja cuantos ciudadanos realizaron algún trámite exitoso en el periodo comprendido entre julio 2006 y enero 2009. Ello permite conocer la sección electoral de residencia del ciudadano y el módulo (fijo, semifijo o móvil) al que acudió. Se parte de la idea que la demanda pasada será similar a la futura, de manera que la evolución de la demanda continuaría la misma tendencia que ha tenido en el pasado próximo.

4. Formalización del problema: se elaboraron diversos escenarios a través de la aplicación de diferentes modelos de localización-asignación implementados en el software ArcInfo Workstation 9.3.

4.1. Matemática: Los modelos considerados fueron:

a) MinDistance: modelo p-mediano que minimiza la impedancia total ponderada de los desplazamientos.

b) MaxAttend: modelo que maximiza la demanda cubierta asumiendo que dicha demanda decae linealmente en función de la distancia/tiempo del desplazamiento.

c) MaxCover: modelo de máxima cobertura que maximiza la demanda cubierta. ${ }^{4}$

4.2. Geográfica: se hace uso de un modelo espacial vectorial dentro del cual dos elementos básicos son considerados:

4.2.1. La red de vías de comunicación (segmentos y nodos) $)^{5}$ proporcionada por el IFE (IFE-RFE, 2008c), la cual incluye como atributo una velocidad promedio para cada segmento, calculándose a partir de dicha velocidad y

4 Para la formulación específica de cada algoritmo véase ESRI (2008), en concreto el apartado Working with linear features. Location-allocation. Model formulation.

5 Una red puede ser definida como un conjunto interconectado de arcos que representan posibles rutas de desplazamientos de recursos de un lugar a otro (ESRI, 2008). 
de la longitud del segmento el tiempo de desplazamiento. Las velocidades asignadas a los distintos tipos de vías de comunicación se muestran en la Tabla 3, alcanzando dicha red un total de 240 mil segmentos con una longitud de 94 mil kilómetros.

Tabla 3

VELOCIDAD POR TIPO DE VÍA $(K M / H)$

\begin{tabular}{|l|r|r|r|r|r|r|}
\hline \multirow{2}{*}{ Tipo de vía } & \multirow{2}{*}{ Promedio } & \multirow{2}{*}{ Máxima } & \multirow{2}{*}{ Mínima } & \multirow{2}{*}{$\begin{array}{c}\text { Desviación } \\
\text { estándar }\end{array}$} & \multicolumn{2}{|c|}{ Segmentos } \\
\cline { 6 - 7 } & & & & \multicolumn{1}{c|}{ Número } & Longitud $(\mathrm{Km})$ \\
\hline Autopista & 80.1 & 90 & 60 & 13.7 & 280 & 691 \\
Pavimentada & 70.2 & 100 & 40 & 13.4 & 2,281 & 4,474 \\
Terracería & 39.8 & 70 & 10 & 8.7 & 3,374 & 9,977 \\
Vialidad & 29.4 & 80 & 1 & 8.7 & 219,208 & 19,764 \\
Brecha & 18.1 & 50 & 5 & 8.7 & 9,756 & 38,853 \\
Vereda & 6.1 & 40 & 3 & 3.1 & 5,571 & 20,474 \\
\hline
\end{tabular}

Fuente: Elaboración propia sobre la base de: IFE-RFE, 2008c.

Figura 1

TIEMPO ESTIMADO DEL RECORRIDO REALIZADO POR LOS CIUDADANOS DESDE SU SECCIÓN DE RESIDENCIA HASTA UN MÓDULO FIJO

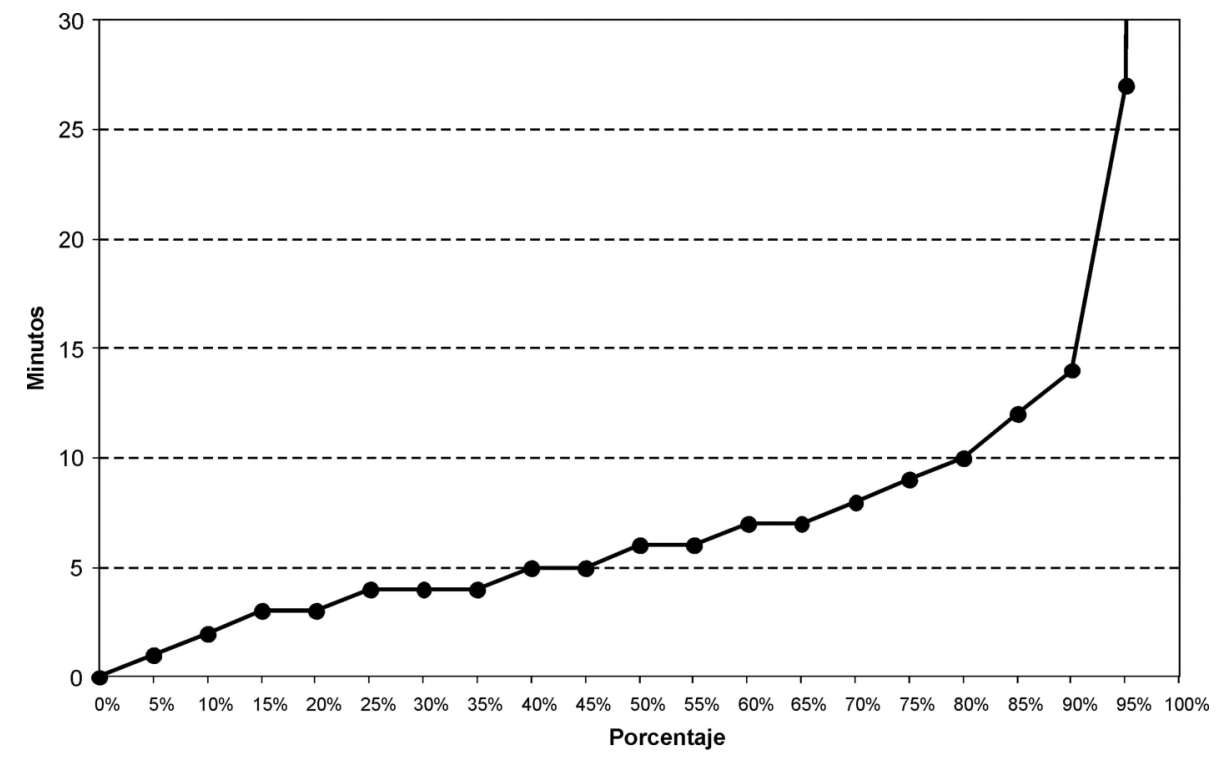

Fuente: Elaboración propia sobre la base de: IFE-RFE, 2008b, 2008c, 2009b. 
Para el cálculo del tiempo máximo de desplazamiento se consideraron los desplazamientos reales de los ciudadanos que se trasladaron para realizar algún trámite en un módulo fijo en el periodo ya señalado, estimándose a través de la red vial sus tiempos de desplazamientos desde el centroide de su sección de residencia hasta el módulo fijo al que acudieron. Como se observa en la Figura 1 el recorrido para el 95\% de estos ciudadanos se estimó en 27 minutos, estimación que se redondeó a 30 minutos, lo que supone el $95.4 \%$ de estos desplazamientos, criterio que fue adoptado como impedancia máxima. Es necesario reflexionar sobre el hecho de que dado que se emplea la velocidad promedio para estimar el tiempo del traslado, ello parece presuponer un desplazamiento en algún tipo de vehículo; sin embargo, las rutas consideradas a través de las vías de comunicación empleadas son rutas aproximadas, dado que se desconoce la ruta exacta empleada por los ciudadanos o el medio de transporte utilizado. Esto implica que este tiempo máximo de recorrido es una abstracción de los tiempos reales, pudiendo darse el caso que un ciudadano, residente dentro de estos 30 minutos de desplazamientos, se haya desplazado a pie y empleado un tiempo mayor a estos 30 minutos.

El uso de esta impedancia máxima supone que el modelo MinDistance aplicado corresponde al denominado p-mediano con restricción de desplazamiento máximo, mientras que en el modelo MaxAttend la constante empleada de decaimiento de la demanda en función de la distancia fue de 1/30 minutos.

4.2.2. Los centroides (puntos) de las secciones electorales. La demanda, así como las posibles ubicaciones de la oferta (módulo fijo) se establecen a partir de los centroides de las secciones electorales (IFE-RFE, 2008b). La ubicación de estos centroides se realizó como sigue a fin de paliar las diferencias en sus tamaños y minimizar posibles problemas vinculados al denominado problema de la unidad espacial modificable (MAUP-modifiable areal unit problem)

4.2.2.1. Para cada sección se consideraron como candidatos a ser seleccionados como centroides las localidades no amanzanadas (puntos).

4.2.2.2. También se consideraron como candidatos aquellos conjuntos de manzanas (polígonos) pertenecientes a una misma localidad, generándose para cada uno de estos conjuntos de manzana un centroide (punto).

4.2.2.3. Para cada sección se seleccionó como ubicación de su centroide aquel punto que contenía un mayor número de ciudadanos empadronados de acuerdo con IFE-RFE (2008d), de manera que el centroide quedó inicialmente localizado en el punto con mayor demanda potencial.

A cada centroide le corresponde una determinada demanda, esto es, la cantidad de ciudadanos de la sección que realizó algún trámite en el periodo ya mencionado. 


\section{ANÁLISIS DE LOS RESULTADOS OBTENIDOS Y POSIBLES ESCENARIOS}

Antes de comparar los resultados obtenidos con respecto de la situación actual de los módulos fijos, es necesario reseñar la diferencia entre la cobertura actual real que estas localizaciones proporcionaron y la cobertura potencial que pudieron haber ofrecido. Por cobertura real se entiende los ciudadanos que de hecho acudieron a estos módulos, mientras por cobertura potencial los ciudadanos que se ubican a un determinado intervalo de tiempo de estos módulos (véase Tabla 4). Los resultados ofrecidos por los modelos aplicados consideran la cobertura potencial, pero resulta imposible conocer, a priori, cual sería su cobertura real, pues como demuestran los desplazamientos realmente desarrollados, no siempre los ciudadanos se desplazaron al módulo más próximo a su residencia, recorriendo incluso un reducido número de ellos grandes distancias; basta comparar el tiempo promedio de desplazamiento entre la cobertura actual real y potencial para una cobertura de hasta 852 mil ciudadanos: mientras los desplazamientos reales obtienen un promedio de casi una hora, el valor para los desplazamientos potenciales sería de únicamente 6 minutos (Tabla 4).

Del análisis de los modelos aplicados, de acuerdo a las restricciones impuestas, (Tabla 4) es posible apreciar que los tres modelos contribuyen a incrementar la demanda potencialmente atendida, por lo que constituyen una mejora respecto de los emplazamientos actuales. No solamente se amplía la cobertura dentro del tiempo máximo establecido ( $\leq 30$ minutos), sino en todos los intervalos de tiempo considerados, aunque esta mejora disminuye en los intervalos menos restrictivos. El modelo MaxCover logra, como es lógico, la mayor cobertura en relación al resto de modelos propuestos.

Si se compara con la cobertura actual real, este incremento en la demanda atendida se ve incluso acompañada por un menor tiempo promedio en todos los modelos dentro de la impedancia máxima fijada (hasta 30 minutos), aunque no si se confronta con la cobertura actual potencial. Estos menores tiempos promedio son particularmente notables en el modelo MaxAttend en comparación con los otros dos modelos, circunstancia que se explica por proponer ubicar los módulos en las zonas de mayor densidad de la demanda, pero a cambio de penalizar en mayor medida a los residentes en zonas más alejadas de los módulos propuestos por este modelo, lo que se refleja en un mayor tiempo promedio, tiempo total y tiempo total ponderado si estos módulos hubieran de atender toda la demanda (75.6 minutos, 153.3 días y 35,880 días).

Como se mencionó, el tiempo promedio total de los desplazamientos que realmente se produjeron hacia los módulos fijos (56.4 minutos) es mucho mayor que el de los desplazamientos potenciales que estas mismas localizaciones pudieron haber producido (5.9 minutos) para atender esa misma demanda (los algo más de 852 ciudadanos). No obstante, todos los modelos analizados ofrecen un mejor tiempo promedio para atender a la demanda total (algo más de 1 millón): frente a los 84.6 minutos de la cobertura actual potencial, el modelo MaxCover ofrece una reducción de casi un 25\% hasta los 64.2 minutos.

Los tiempos totales (no ponderados) muestran nuevamente una similitud entre los modelos MinDistance y MaxCover, la superioridad del modelo MaxAttend para atender la demanda más próxima a los módulos propuestos por este modelo, y la notoria mejora ofrecida por los tres modelos en relación con la cobertura actual real, hecho que confirman los 

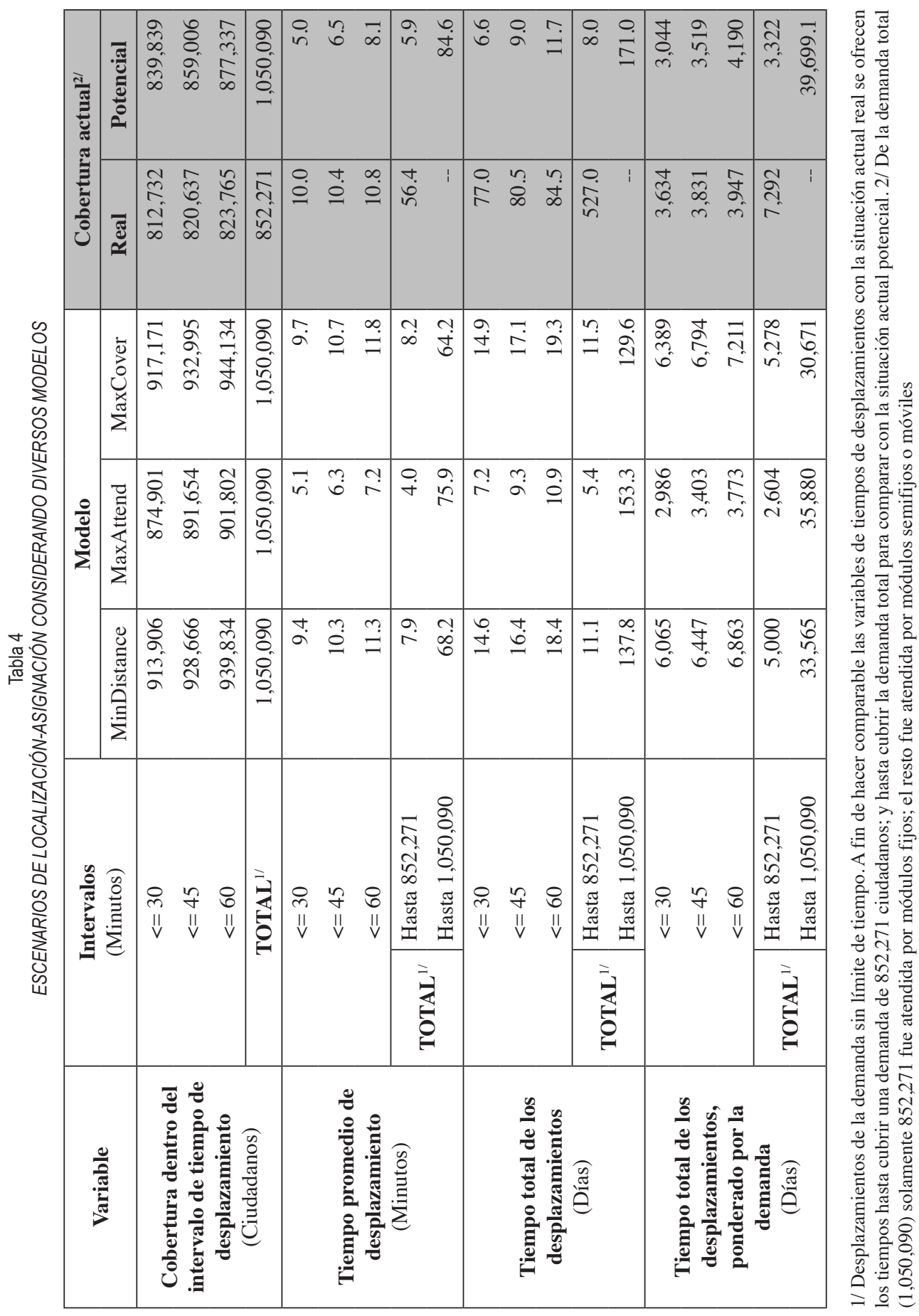
tiempos totales ponderados. ${ }^{6}$ Considerando la demanda total (un millón), los tres modelos suponen un avance en esta variable respecto de la cobertura actual potencial, aunque únicamente el modelo MaxAttend obtiene superiores resultados para un cubrimiento de 852 mil ciudadanos, escenario que se repite en la variable tiempo total ponderado.

En ambos tiempos totales (ponderado y no ponderado), el modelo MinDistance supera al modelo MaxCover en los desplazamientos de hasta 60 minutos, pero no si los módulos propuestos han de cubrir la demanda total, en cuyo caso la situación se invierte, imponiéndose el modelo MaxCover al modelo MinDistance.

En definitiva, los modelos propuestos constituyen una mejora respecto de la cobertura actual real: permiten un aumento del cubrimiento de la demanda (también para la cobertura actual potencial), y similares o menores tiempos promedio de desplazamiento, tiempos promedio que disminuyen de manera notable a medida que aumenta la demanda cubierta si se compara con la cobertura actual real. No obstante la comparación no es ventajosa para los modelos propuestos (MinDistance y MaxCover) en cuanto a tiempos promedios para la demanda más próxima si se confronta con la cobertura actual potencial. Esta circunstancia se repite en la variable tiempos totales. El modelo MaxAttend claramente ofrece una mejor localización para la atención de la demanda más cercana a los módulos propuestos (menor tiempo promedio y tiempos totales), pero a costa de un menor cubrimiento y de penalizar a los ciudadanos más alejados de los módulos sugeridos. Mientras, los modelos MinDistance y MaxCover obtienen resultados similares en cuanto a cobertura y tiempos: ligera mayor cobertura en MaxCover y algo menores tiempos en MinDistance, aunque si se analizan los tiempos para la demanda total (algo más de un millón de ciudadanos) el modelo MaxCover arroja mejores datos.

Dada la cobertura universal que debe ofrecer el IFE en cuanto a la provisión de credenciales, el hecho de que el modelo MaxAttend supondría una mayor discriminación espacial en términos de desplazamientos para ciertos ciudadanos, y de que el modelo MaxCover ofrece no sólo la mayor cobertura sino también los menores tiempos de desplazamiento (promedio, total y total ponderado) para la totalidad de la demanda, se considera a dicho modelo como el más adecuado.

A través de la Figura 2 es fácil apreciar como la distribución propuesta es ciertamente diferente a la existente. Básicamente, la aplicación del algoritmo propone, a fin de maximizar la cobertura, reducir la presencia de módulos fijos en las ciudades más grandes de la entidad (Ciudad Juárez y Chihuahua), a cambio de sustituirlos por un número más reducidos pero de mayor tamaño/capacidad. La mayor facilidad para el transporte, y por tanto los menores tiempos de desplazamientos en las grandes ciudades, justifican esta solución.

En conjunto la nueva distribución de módulos permitiría incrementar el número de ciudadanos ubicados hasta a 30 minutos de un módulo fijo de casi 840 mil a 917 mil, lo que supone una aumento del 9.2\% (Tabla 4). Ello implicaría una mejora desde el punto de vista de la justicia espacial en términos de incrementar la accesibilidad a dichos módulos fijos a un mayor número de ciudadanos.

6 Dado que la demanda varía en cada modelo y en cada intervalo de tiempo, el dato estrictamente comparable entre los diferentes modelos y la situación actual real y potencial, corresponde al tiempo total (ponderado y no ponderado) hasta 825,271 y $1,050,090$ ciudadanos. 
Figura 2

MÓDULOS DE ATENCIÓN CIUDADANA FIJOS EXISTENTES Y PROPUESTOS (ESCENARIO MAXCOVER1)
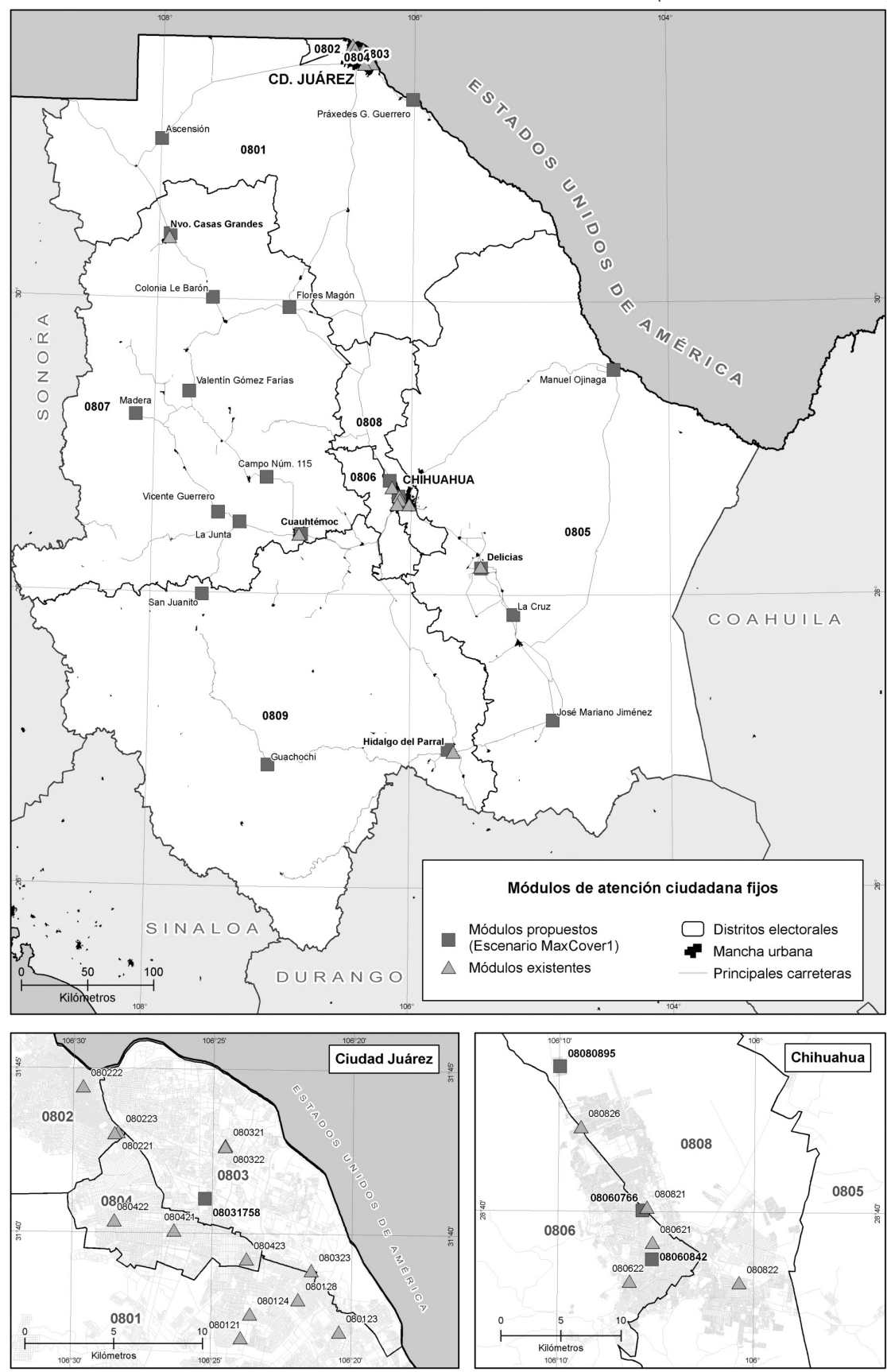

Fuente: Elaboración propia a partir de: IFE-RFE, 2008b, 2008c, 2009a 


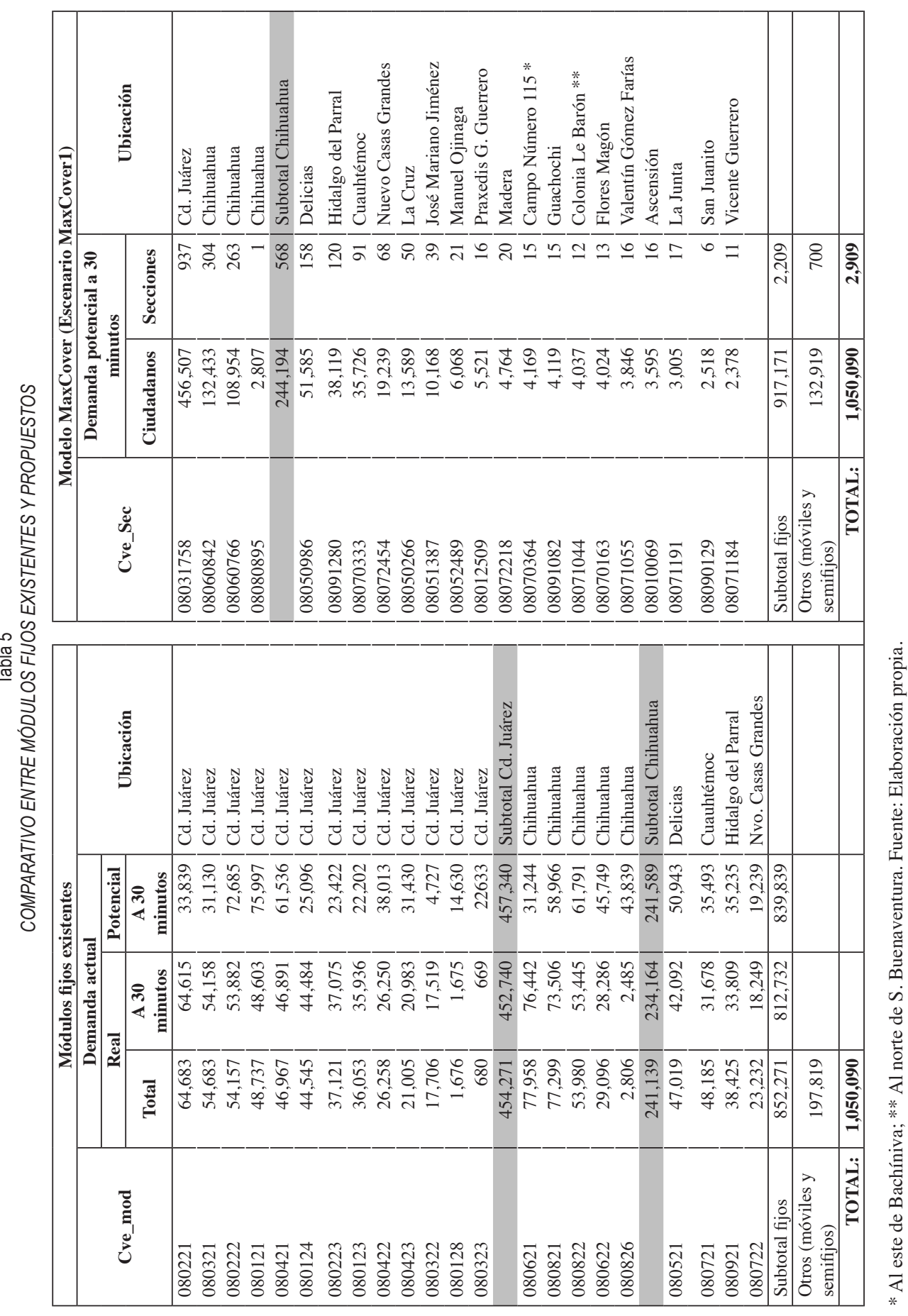


En Ciudad Juárez el número de módulos fijos se reduce de trece a únicamente uno, lo que supone una ligera reducción (algo más de 800 ciudadanos) de la demanda actual potencial situada a 30 minutos frente al alcanzado por el emplazamiento sugerido. Por el contrario, en la ciudad de Chihuahua las localizaciones sugeridas permitirían un aumento de la demanda potencial atendida a 30 minutos de algo más de 2,500 ciudadanos, pese a la reducción en el número de módulos fijos de cinco a sólo tres. ${ }^{7}$

En ambas ciudades las ubicaciones propuestas resultan próximas a ciertos módulos ya existentes, por lo que una ampliación de los mismos surge como una posible solución, sobre todo si se considera el pequeño tamaño de las secciones electorales en las grandes áreas urbanas, por lo que una localización próxima a las propuestas tendría un escaso impacto en la demanda a atender, aunque obviamente se trataría de un sub-óptimo en relación al modelo original. Este aspecto se desarrolla posteriormente. En este sentido, la creación de macromódulos era una idea ya existente en el IFE antes de realizar este análisis, por lo que su implementación debería tener escasos obstáculos al interior de la institución.

También muy próxima a la ubicación actual se sitúan los módulos propuestos para Cuauhtémoc, Delicias, Hidalgo del Parral y Nuevo Casas Grandes (Figura 3.C y 3.D), aunque su reubicación permitiría una mayor cobertura, sobre todo en el caso de Hidalgo del Parral, donde la mejora llega al 8.2\% sobre la demanda potencial a 30 minutos. La nueva ubicación propuesta para el módulo de Nuevo Casas Grandes obtendría el mismo cubrimiento a 30 minutos que la demanda actual potencial, por lo que su ganancia sería nula.

Ciertos módulos fijos existentes destacan por haber cubierto una demanda real muy por debajo de la potencial: en Cd. Juárez los módulos 080323 y 080128 ; en Chihuahua los módulos 080826 y 080622 o el módulo de Delicias; en una situación opuesta, mayor demanda real que potencial, se encuentran otros módulos existentes, por ejemplo el módulo 080221 de Cd. Juárez. Todo ello pone de manifiesto el comportamiento «no óptimo/racional» de la población al no desplazarse al módulo más cercano a su residencia, sino mostrar una preferencia por ciertos módulos en detrimento de otros.

Una novedad de la propuesta ofrecida surge de la reubicación de los catorce módulos fijos excedentarios de las dos principales ciudades del estado. Como ya se ha mencionado, ello incrementaría la cobertura total, favoreciendo su accesibilidad a casi 72 mil ciudadanos que realizarían desplazamientos de no más de 30 minutos. Esta circunstancia beneficiaría a zonas menos urbanizadas de distintas áreas de la entidad federativa, tanto en la zona fronteriza (módulos de Práxedes G. Guerrero y Manuel Ojinaga), como en el sur (José Mariano Jiménez y Guachochi) y occidente (San Juanito, La Junta y Colonia Le Barón, entre otros) (Figura 2). Muy probablemente la reubicación de módulos fijos en estas localidades atraería a ciudadanos ubicados a más de los 30 minutos establecidos como óptimo, dado que a partir del análisis de la ubicación de la demanda atendida en módulos no situados en Cd. Juárez y Chihuahua, se pudo constatar que dichos módulos tienen una cobertura espacial más amplia.

La Tabla 6 muestra como los distritos menos beneficiados por la relocalización propuesta serían el 0801, 0802 y 0804, todos ellos afectados por la pérdida de módulos de

7 En ambas localidades el modelo MinDistance propone ubicar un único módulo fijo, mientas el modelo MaxAttend formula el establecimiento de ocho módulos fijos en Cd. Juárez y cinco en Chihuahua. Ambos modelos coinciden con el modelo MaxCover en situar un módulo fijo en las ciudades de Delicias, Cuauhtémoc, Nvo. Casas Grandes e Hidalgo del Parral. 
Ciudad Juárez, aunque en el caso del distrito 0801 se proponen dos nuevos módulos en Práxides G. Guerrero y Ascensión. En estos tres distritos la pérdida de demanda atendida sería cubierta por el módulo ubicado en Ciudad Juárez (distrito 0803), el cual tendría que cubrir la demanda existente en toda la ciudad. Respecto a esta disminución en el número de módulos de Ciudad Juárez, cabe mencionar que en la actualidad dos módulos se encuentran muy próximos a otros dos, a menos de 300 metros de distancia, mientras otros dos módulos cubren una demanda muy baja (véase Tabla 5), todo lo cual justifica su reducción.

Algo similar sucede con la demanda del distrito 0808, la cual sería atendida por los módulos ubicados en el distrito 0806, en la ciudad de Chihuahua.

Tabla 6

COMPARATIVO POR DISTRITO ENTRE MÓDULOS FIJOS EXISTENTES Y PROPUESTOS (ESCENARIO MAXCOVER1)

\begin{tabular}{|c|c|c|c|c|}
\hline \multirow{3}{*}{ Distrito } & \multirow{3}{*}{$\begin{array}{l}\text { Módulos } \\
\text { existentes }\end{array}$} & \multicolumn{3}{|c|}{ Demanda actual } \\
\hline & & \multicolumn{2}{|c|}{ Real } & \multirow{2}{*}{$\begin{array}{c}\text { Potencial } \\
\text { A } 30 \\
\text { minutos (a) }\end{array}$} \\
\hline & & Total & $\begin{array}{c}\text { A } 30 \\
\text { minutos }\end{array}$ & \\
\hline 0801 & 4 & 131,011 & 130,698 & 137,925 \\
\hline 0802 & 3 & 155,961 & 155,572 & 129,946 \\
\hline 0803 & 3 & 73,069 & 72,346 & 58,490 \\
\hline 0804 & 3 & 94,230 & 94,124 & 130,979 \\
\hline 0805 & 1 & 47,019 & 42,092 & 50,943 \\
\hline 0806 & 2 & 107,054 & 104,728 & 76,993 \\
\hline 0807 & 2 & 71,417 & 49,927 & 54,732 \\
\hline 0808 & 3 & 134,085 & 129,436 & 164,596 \\
\hline 0809 & 1 & 38,425 & 33,809 & 35,235 \\
\hline TOTAL: & 22 & 852,271 & 812,732 & 839,839 \\
\hline
\end{tabular}

\begin{tabular}{|r|r|r|}
\hline $\begin{array}{c}\text { Módulos } \\
\text { propuestos }\end{array}$ & $\begin{array}{c}\text { Demanda } \\
\text { potencial a } \\
\mathbf{3 0} \text { minutos } \\
\text { (b) }\end{array}$ & $\begin{array}{c}\text { Diferencia } \\
\text { (b)-(a) }\end{array}$ \\
\hline 2 & 9,116 & $-128,809$ \\
0 & -- & $-129,946$ \\
1 & 456,507 & 398,017 \\
0 & -- & $-130,979$ \\
4 & 81,410 & 30,467 \\
2 & 241,387 & 164,394 \\
9 & 81,188 & 26,456 \\
1 & 2,807 & $-161,789$ \\
3 & 44,756 & 9,521 \\
\hline $\mathbf{2 2}$ & $\mathbf{9 1 7 , 1 7 1}$ & $\mathbf{7 7 , 3 3 2}$ \\
\hline
\end{tabular}

Fuente: Elaboración propia.

Mención especial merece la localización propuesta en ciertas poblaciones como La Cruz, Colonia Le Barón o Campo Número 115 (Tabla 5), dado que, si bien se sitúan en secciones con una demanda relativamente importante $(318,519$ y 691, respectivamente), su posición estratégica les permite atender la demanda de localidades urbanas próximas más relevantes y áreas circundantes, por lo que en términos de extensión territorial su cobertura es mayor que los módulos situados en ciudades grandes o medias (véase Figura 3.E y 3.F).

En definitiva, los emplazamientos propuestos permitirían que el porcentaje de secciones atendidas ascendiera al $75.94 \%$, cifra que alcanza el $87.34 \%$ en términos de ciudadanos atendidos. Únicamente el $13.46 \%$ de estos ciudadanos se ubicaría a más de 30 minutos de los módulos fijos planteados (Tabla 7), caracterizándose por su gran dispersión espacial, por lo que su atención quedaría principalmente en manos de módulos semifijos y móviles. 
Tabla 7

COBERTURA DE LOS MÓDULOS FIJOS PROPUESTOS A 30 MINUTOS (ESCENARIO MAXCOVER1)

\begin{tabular}{|l|r|r|r|r|}
\hline & \multicolumn{2}{|c|}{ Secciones } & \multicolumn{2}{c|}{ Demanda } \\
\cline { 2 - 5 } & Núm & \% & Núm & \multicolumn{1}{c|}{$\%$} \\
\hline Atendidas & 2,209 & 75.94 & 917,171 & 87.34 \\
No atendidas & 700 & 24.06 & 132,919 & 12.66 \\
\hline Total & 2,909 & 100.00 & $1,050,090$ & 100.00 \\
\hline
\end{tabular}

Fuente: Elaboración propia.

A continuación se plantean tres escenarios alternativos al modelo original (MaxCover1) en aras a mantener los módulos fijos ya existentes. El escenario MaxCover2a presenta una mayor fidelidad respecto del modelo original (MaxCover1), mientras el escenario MaxCover3 trata de conservar el status quo actual; por último, el escenario MaxCover2b plantea una situación intermedia. Las características específicas de estos escenarios son:

1. MaxCover1: escenario original planteado de acuerdo a las restricciones ya descritas.

2. MaxCover2a: dado que el escenario MaxCover1 propone situar módulos fijos en ciudades donde ya existen algunos de estos módulos, se establece que su emplazamiento coincida con módulos ya existentes en dichas ciudades, en concreto con aquellos módulos que cubrieron una mayor demanda real en el periodo julio 2006enero 2009. De acuerdo a este escenario los módulos fijos de Cuauhtémoc, Delicias, Hidalgo del Parral y Nvo. Casas Grandes mantendrían su ubicación actual, mientras en las ciudades de Juárez y Chihuahua conservarían su localización únicamente uno y tres módulos respectivamente, los de mayor demanda real a 30 minutos (véase Tabla 5).

3. MaxCover2b: mismo escenario que MaxCover2a pero asignando un módulo adicional a Cd. Juárez a fin de moderar la drástica reducción de módulos en esta ciudad y evitar así la concentración de toda su demanda en un único módulo fijo. Esto supone conservar los dos módulos fijos con mayor demanda real a 30 minutos en esta ciudad y la supresión del módulo de Vicente Guerrero a fin de mantener el número de módulos fijos en 22 .

4. MaxCover3: mismo número de módulos fijos en las mismas ciudades de acuerdo con su ubicación actual, variando únicamente su emplazamiento al interior de dichas ciudades.

Los escenarios MaxCover2a, MaxCover2b y MaxCover3 constituyen sub-óptimos del escenario original, MaxCover1, lo que explica una cobertura algo inferior; a cambio, disminuyen los tiempos promedio de los recorridos, al menos en los intervalos de hasta 60 minutos (Tabla 8). Pese a ello, la demanda atendida en los nuevos tres escenarios continua siendo superior a la cobertura actual potencial, a costa siempre de mayores desplazamientos promedios en la demanda más próxima a los módulos. En los cuatro escenarios MaxCover el desplazamiento promedio para la demanda total surge como un elemento positivo en relación con la cobertura actual potencial, hecho que se repite para las variables tiempo total y tiempo total ponderado, excepción hecha del escenario MaxCover3. En función de ello, este último escenario parece el menos adecuado, pues si bien supone una ligera mayor cobertura que la 


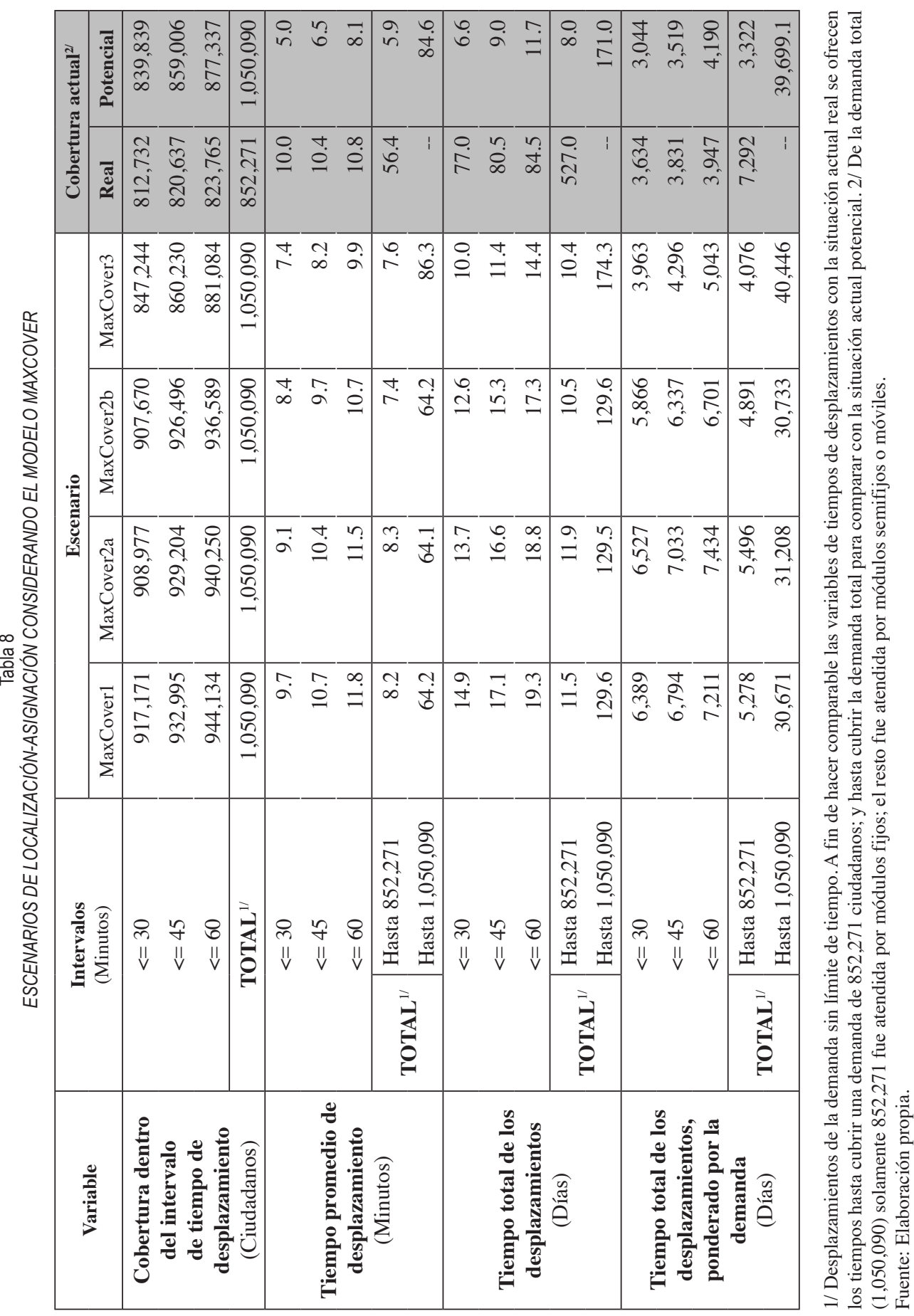


actual potencial, los usuarios más alejados de los módulos propuestos se verían más penalizados en sus tiempos de desplazamiento.

De los escenarios expuestos quizás el más realista sea MaxCover2b: si bien la ganancia en la demanda cubierta es algo inferior a los escenarios MaxCover1 y MaxCover2a, supone en general menores tiempos de desplazamiento, tanto promedio como totales y totales ponderados. Implica también un mejor aprovechamiento de la infraestructura existente y, en el caso de Cd. Juárez, una reducción menos drástica en cuanto al número de módulos fijos en esta ciudad.

\section{CONCLUSIONES Y RECOMENDACIONES}

El presente trabajo es una muestra de las aportaciones que desde la Geografía se pueden realizar en diversos ámbitos, en este caso la localización de instalaciones públicas con fines electorales, mostrando las posibilidades de colaboración entre la Geografía y el Instituto Federal Electoral (IFE). Es a través de este tipo de colaboraciones que la utilidad del conocimiento y técnicas geográficas ya consolidadas como los modelos de localización-asignación pueden permear al interior de estas instituciones, fomentando su difusión y valoración por otros profesionales no geógrafos. En este sentido, este estudio piloto en el estado de Chihuahua despertó el suficiente interés en el IFE como para replicar el análisis en otras entidades del país, planteando el debate sobre qué tan óptima es la localización actual de los módulos fijos.

Los emplazamientos propuestos de módulos fijos, a través de diferentes algoritmos y escenarios, responden a la distribución espacial de la demanda y, por tanto, de la población, la cual presenta una importante concentración en los dos principales núcleos urbanos de la entidad, Ciudad Juárez y Chihuahua. La estrategia ofrecida propone una reducción del número de módulos fijos en estos núcleos urbanos pero a cambio de incrementar su capacidad, lo que se justifica por la alta accesibilidad al interior de estas ciudades si se compara con el resto del estado; la creación de macro-módulos ya era una posibilidad considerada por el propio IFE antes de este estudio. Como contrapartida se obtiene una mayor equidad espacial en el reparto de y acceso a estos módulos fijos, lo cual beneficia principalmente a ciudades medias y pequeñas del estado, cuya posibilidad de responder a la demanda de las áreas rurales aledañas es relevante.

Sin embargo, el modelo de localización aplicado se basa en una serie de supuestos y datos que deben ser tomados con cierta cautela a la hora de evaluar las localizaciones propuestas. Dos son los insumos básicos del modelo: la ubicación de la demanda y la accesibilidad de los potenciales lugares de ubicación (secciones) de la oferta (módulos), accesibilidad medida en términos de tiempos de recorrido a través de una red vial. ${ }^{8}$ Respecto a la ubicación de la demanda la suposición de que la demanda futura sea similar a la del pasado reciente puede o no ser cierta; por ejemplo, la redistribución de la demanda a partir de movimientos migrato-

8 En relación a los estudios aplicados de localización de instalaciones Current et al. (1990) señalan como la mayoría están relacionados con la minimización de costos, generalmente asociados a la reducción de costes de transportes a través de la consideración de las distancias, aunque otras categorías de interés señaladas son la localización de instalaciones al objeto de optimizar la demanda/población atendida (opción elegida en este estudio), aquellas que buscan maximizar el beneficio y las vinculadas con preocupaciones medioambientales. 
Figura 3

EJEMPLOS DE DEMANDA ASIGNADAA MÓDULOS FIJOS PROPUESTOS (ESCENARIO MAXCOVER2B)
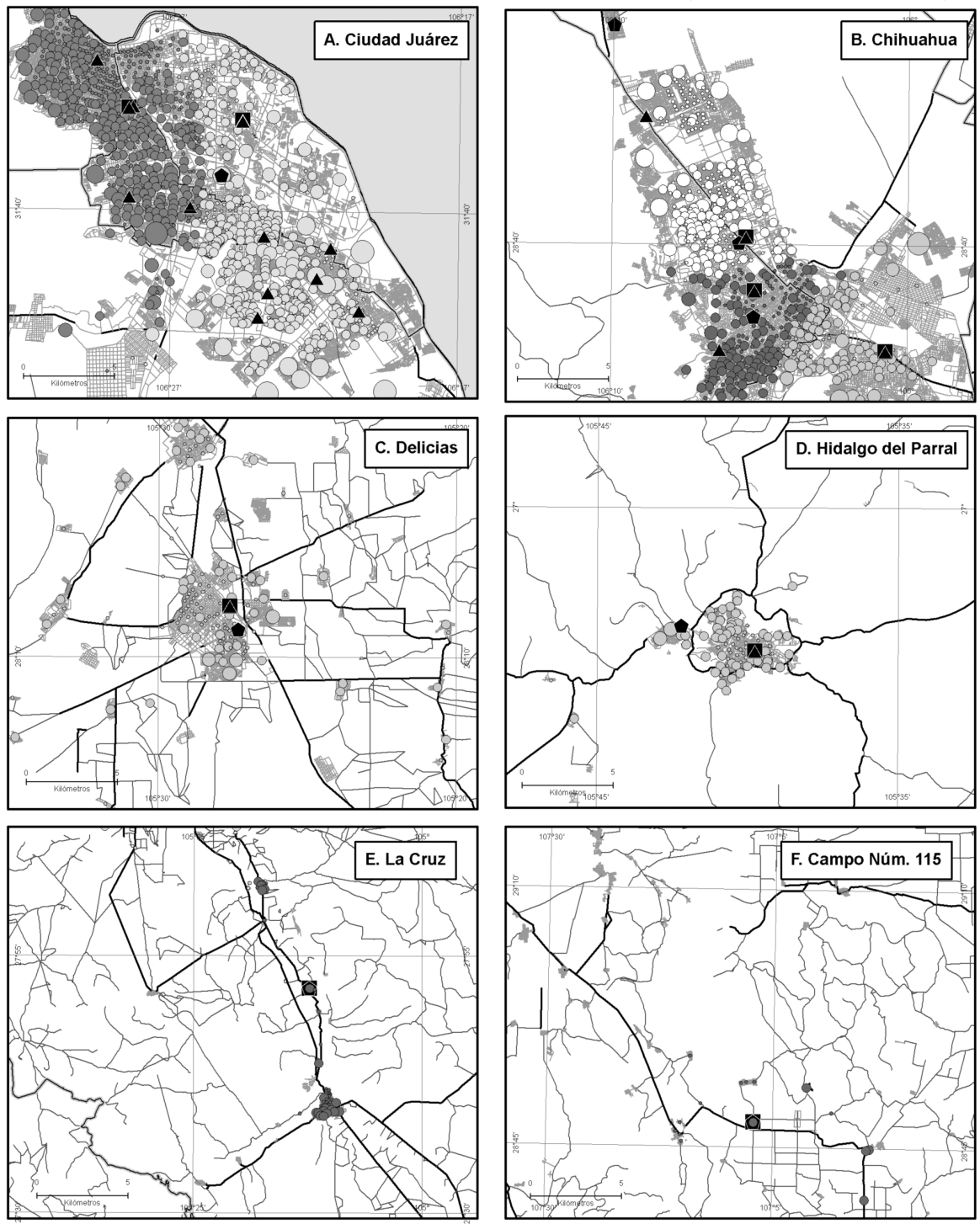

\section{Módulos fijos}

$\Delta$ Existentes

Propuestos:

- Escenario MaxCover1

Escenario MaxCover2b

Fuente: Elaboración propia.
Cobertura: demanda atendida por sección (a 30 minutos; escenario MaxCover2b)

$$
<=300
$$

- $301-1,000$

- $1,001-2,500$

- $2,501-6,000$

$6,001-11,996$

\section{Vías de comunicación}

- Autopista y pavimentada Calles

_ Terracería, brecha o vereda

Límites de distritos 
rios alteraría su ubicación, aunque un drástico cambio en la distribución de la población no parece probable a corto o mediano plazo.

En relación a la accesibilidad, ésta es también susceptible de modificarse, por ejemplo, mediante la construcción y/o mejora de vías de comunicación. Dada la relevancia de este insumo en el resultado final, cabe mencionar los problemas de precisión, actualización y conectividad en la cartografía vial ofrecida por el IFE, pese a ser una de las más completas disponibles a nivel intra-urbano. Ello se justifica por el hecho de que dicha cartografía vial tiene como fin principal permitir al ciudadano ubicar su lugar de residencia a la hora de gestionar su credencial de elector, por lo que no fue concebida para realizar análisis de redes. El hecho de que otras instituciones públicas también desarrollen su propia cartografía vial, por ejemplo la Secretaría de Comunicaciones y Transportes (SCT) y el Instituto Nacional de Estadística, Geografía e Informática (INEGI), hace aconsejable la colaboración entre estas instituciones en aras a evitar la duplicidad del gasto; también recomendable es la colaboración con empresas privadas (p.e., NAVTEQ) dedicadas a la elaboración de sistemas de navegación vía GPS a fin de obtener una mejor cartografía vial. Otras opciones para obtener tiempos de desplazamientos lo más reales posibles (o al menos de validar los aquí estimados) consistiría en encuestar a los ciudadanos que acuden a los módulos fijos sobre sus desplazamientos, o mejorar la velocidad asignada a los tramos de determinadas vías de comunicación en función del conocimiento empírico de sus residentes. Este aspecto es especialmente importante al interior de grandes ciudades, donde la congestión vial es un factor determinante en los tiempos de desplazamientos.

Finalmente es necesario mencionar las variables no incluidas en el modelo. Entre ellas sobresalen los posibles costos financieros de reubicación de los módulos y los efectos a corto plazo sobre los ciudadanos, conocedores y acostumbrados a acudir a los módulos existentes. Cabe también recordar que el análisis, así como la propuesta de ubicación de módulos fijos se realizó a nivel de sección, secciones que son relativamente pequeñas en el caso de las ciudades; ello y la proximidad de algunas de las localizaciones propuestas a módulos ya existentes sugiere la posibilidad de ampliar y/o mantener algunos de estos módulos, aspecto valorado a través de la elaboración de algunos escenarios. Así, los emplazamientos propuestos deben considerarse como una primera aproximación, la cual debe ser valorada dentro de un marco de discusión más amplio donde el conocimiento empírico de las representaciones locales del IFE debe desempeñar un papel relevante.

\section{REFERENCIAS BIBLIOGRÁFICAS}

ALONSO, W. (1964): Location and land use: toward a general theory of land rent, Massachusetts: Harvard University Press.

BOSQUE, J. y MORENO, A. (2004): «La localización óptima como problema: cuestiones teóricas y metodológicas», en Sistemas de Información Geográfica y localización de instalaciones y equipamientos (Bosque, J. y Moreno, A., coords.), Madrid: Ra-Ma, 3-16.

BOSQUE, J., GÓMEZ, M. y PALM, F. (2006): «Un nuevo modelo para localizar instalaciones no deseables: ventajas derivadas de la integración de modelos de localizaciónasignación y SIG», Cuadernos Geográficos, núm. 36, pp. 53-68. 
BOSQUE, J. y MORENO, A. (2007): «Localización-asignación y justicia/equidad espacial con Sistemas de información geográfica», Memorias de la XI Conferencia Iberoamericana de SIG, Lujan, 95-115. Disponible en http://www.geogra.uah.es/inicio/web_11_ confibsig/SEMINARIO/LS_Bosque_Moreno.pdf.

BUZAI G.D. y BAXENDALE, C.A. (2008): «Modelos de localización-asignación aplicados a servicios públicos urbanos: análisis espacial de escuelas EGB en la ciudad de Luján», en Análisis y planificación de servicios colectivos con Sistemas de Información Geográfica (Moreno, A. y Buzai, G.D., coords.), Madrid: Universidad Autónoma de Madrid, 95-116.

CHRISTALLER, W. (1933): Die zentralen orte in Süddeutschland, Jena: Gustav Fischer.

CHURCH, R. y REVELLE, Ch. (1974): «The maximal covering location problem», Papers of the Regional Science Association, vol. 32, 101-118.

CURRENT, J., MIN, H. y SCHILLING, D. (1990): Multiobjective analysis of facility location decisions, European Journal of Operational Research, núm. 49, 295-307.

DASKIN, M.S. (2008): «What you should know about location modeling», Naval Research Logistics, vol. 55, núm. 4, 283-294.

ESRI / Environmental Systems Research Institute (2008): ArcDoc Version 9.3, Redlands: ESRI.

GARROCHO, C. (1993): «Eficiencia, igualdad y equidad en la localización de los servicios de salud infantil del Estado de México», Estudios Demográficos y Urbano, vol. 8, núm. 3, 601-640.

HAGGETT, P. (1965): Location analysis in Human Geography, London: Edward Arnold.

IFE-RFE / Instituto Federal Electoral-Registro Federal de Electores (2005): Tipología de los distritos electorales para la aplicación de las actividades de la DERFE (Archivo interno).

IFE-RFE / Instituto Federal Electoral-Registro Federal de Electores (2008a): Verificación Nacional Muestral. Informe Final (Archivo interno)

IFE-RFE / Instituto Federal Electoral-Registro Federal de Electores (2008b): Cartografía geoelectoral del estado de Chihuahua, Noviembre. Cartografía digital (Archivo interno)

IFE-RFE / Instituto Federal Electoral-Registro Federal de Electores (2008c): Rasgos cartográficos de referencia del estado de Chihuahua, Noviembre. Cartografía digital (Archivo interno).

IFE-RFE / Instituto Federal Electoral-Registro Federal de Electores (2008d): Padrón electoral del estado de Chihuahua a 31 de diciembre de 2008 (Archivo interno).

IFE-RFE / Instituto Federal Electoral-Registro Federal de Electores (2009a): Reporte de avance del operativo por módulo SIRFE. 3 de julio de 2006-15 de enero de 2009 (Archivo interno).

IFE-RFE / Instituto Federal Electoral-Registro Federal de Electores (2009b): Solicitudes exitosas en módulos del Instituto Federal Electoral. Periodo: de 6 julio de 2006 a 15 de enero 2009 (Archivo interno).

ISARD, W (1956): Location and space-economy. A general theory relating to industrial location, market areas, land use, trade, and urban structure, Cambridge: Massachusetts Institute of Technology-Wiley. 
ISARD, W. (1960): Methods of regional analysis. An introduction to regional science, Cambridge: Massachusetts Institute of Technology-Wiley.

ISARD, W. (1998): «Gravity and spatial interactions models», en Methods of interregional and regional analysis (Isard, W., Azis, I.J., Drennan, M.P., Miller, R.E., Saltzman, S. y E. Thorbecke, eds.), Hants: Ashgate, 243-279.

LÖSCH, A. (1940): Die räumliche ordnung der wirtschaft, Jena: Gustav Fischer.

MILLER, J.M. y SHAW, S.L. (2001): Geographic information systems for transportation. Principles and applications, New York: Oxford University Press.

MORENO, A. (2004): «Modelos de localización óptima de instalaciones y equipamientos», en Sistemas de Información Geográfica y localización de instalaciones y equipamientos (Bosque, J. y Moreno, A., coords.), Madrid: Ra-Ma, 53-101.

ROY, J.R. (2004): Spatial interaction modeling, Heidelberg: Springer.

SEDESOL-CONAPO-INEGI / Secretaría de Desarrollo Social-Consejo Nacional de Población-Instituto Nacional de Estadística, Geografía e Informática (2007): Delimitación de las zonas metropolitanas de México 2005, México D.F.

SMITH, H K., LAPORTE, G. y HARPER, P.R. (2009): «Locational analysis: highlights of growth to maturity», Journal of the Operational Research Society, vol. 60, sup. 1, 140148.

VON THÜNEN, J.H, (1826): Der isolirte staat in beziehung auf landwirthschaft und nationalokonomie, oder, Untersuchungen uber den einfluss, den die getreidepreise, der reichthum des bodens und die abgaben auf den ackerbau ausuben, Hamburg : F. Perthes.

ULLMAN, E.L. (1941): «A theory of location for cities», American Journal of Sociology, vol. 46, núm. 6, 853-864.

WANG, F. (2006): Quantitative methods and applications in GIS, Boca Ratón: Taylor \& Francis.

WEBER, A. (1909): Theory of the location of industries. Chicago: University of Chicago Press.

ZEBADÚA, E. (2010): «El rumbo de la Cédula de Identidad Ciudadana», en La Crónica de Hoy, 16 de agosto. Disponible en http://www.cronica.com.mx/nota.php?id_nota=525913. 
